\title{
The presence of postmenopausal bleeding as prognostic parameter in patients with endometrial cancer: a retrospective multi-center study
}

\author{
Veronika Seebacher*1, Maximilian Schmid ${ }^{1}$, Stephan Polterauer ${ }^{1}$, \\ Katrin Hefler-Frischmuth ${ }^{2}$, Heinz Leipold ${ }^{3}$, Nicole Concin ${ }^{4}$, \\ Alexander Reinthaller ${ }^{1}$ and Lukas Hefler ${ }^{1}$
}

Address: ${ }^{1}$ Department of Obstetrics \& Gynecology, Medical University of Vienna, Währinger Gürtel 18-20, 1090 Vienna, Austria, ${ }^{2}$ Department of Laboratory Medicine, Wilhelminenspital, Montleartstraße 37, 1160 Vienna, Austria, ${ }^{3}$ Department of Obstetrics \& Gynecology, Landeskrankenhaus Klagenfurt, St. Veiter Strasse 47, 9020 Klagenfurt, Austria and ${ }^{4}$ Department of Obstetrics \& Gynecology, Innsbruck Medical University, ChristophProbst-Platz, Innrain 52, 6020 Innsbruck, Austria

Email: Veronika Seebacher* - veronika.seebacher@meduniwien.ac.at; Maximilian Schmid - maximilian.schmid@meduniwien.ac.at; Stephan Polterauer - stephan.polterauer@meduniwien.ac.at; Katrin Hefler-Frischmuth - katrin.hefler@gmail.com; Heinz Leipold - dozentleipold@ordination-leipold.at; Nicole Concin - nicole.concin@i-med.ac.at; Alexander Reinthaller - alexander.reinthaller@meduniwien.ac.at; Lukas Hefler - lukas.hefler@meduniwien.ac.at

* Corresponding author

Published: 22 December 2009

BMC Cancer 2009, 9:460 doi:I0.| | 86/|47|-2407-9-460

This article is available from: http://www.biomedcentral.com/I47I-2407/9/460

(C) 2009 Seebacher et al; licensee BioMed Central Ltd.

This is an Open Access article distributed under the terms of the Creative Commons Attribution License (http://creativecommons.org/licenses/by/2.0), which permits unrestricted use, distribution, and reproduction in any medium, provided the original work is properly cited.
Received: 12 February 2009

Accepted: 22 December 2009

\begin{abstract}
Background: To date, there is no consensus on the utility of screening procedures for the early detection of endometrial cancer. The value of transvaginal ultrasound for screening of asymptomatic endometrial cancer has been discussed controversially. This study was conducted to evaluate whether asymptomatic patients with endometrial cancer have a better prognosis than symptomatic patients with endometrial cancer diagnosed after postmenopausal bleeding.
\end{abstract}

Methods: In the present multi-center study, the effect of the presence of postmenopausal bleeding on prognosis was evaluated retrospectively in 605 patients with endometrial cancer using patients' files. 543 patients (I33 patients were asymptomatic, 410 patients were symptomatic) with endometrioid endometrial cancer were enrolled in all further analysis. Student's t-test, Cox regression analysis and Kaplan-Meier analysis were used were appropriate.

Results: Presence/absence of a postmenopausal bleeding was not associated with tumor stage ( $p$ $=0.2$ ) and age at diagnosis $(p=0.5)$. Asymptomatic patients with endometrial cancer had a significantly higher rate of well and moderate-differentiated tumors compared to symptomatic patients $(p=0.008)$. In univariable and multivariable survival analysis, tumor stage, tumor grade, and patients' age at diagnosis, but not presence/absence of a postmenopausal bleeding, were associated with disease free and overall survival.

Conclusion: Asymptomatic patients with endometrial cancer have a higher rate of well differentiated tumors compared to patients with a postmenopausal bleeding prior to diagnosis. The prognosis of both groups of patients was similar. 


\section{Background}

Endometrial cancer is the most common gynecological malignancy in developed countries with an estimated 39,000 newly diagnosed cases in the United States in 2007. Although many patients are diagnosed with an early stage disease, 7400 deaths will occur [1].

Approximately $90 \%$ of women with endometrial cancer have abnormal uterine bleeding as the only presenting complaint leading to the diagnosis of the disease [2]. To date, there is no consensus on the utility of screening procedures for the early detection of endometrial cancer [38]. It has been suggested that screening of asymptomatic endometrial cancer by transvaginal ultrasound before the onset of clinical symptoms, i.e., postmenopausal bleeding, leads to an earlier diagnosis [8]. As in other malignancies, it can be hypothesized that an earlier diagnosis leads to a lower stage of disease, a less radical surgery, a therapy with less side effects and, subsequently, a better prognosis of affected patients.

Interestingly, relatively few studies are available investigating whether asymptomatic patients with endometrial cancer have a better prognosis than those detected after the onset of a postmenopausal bleeding. Gerber et al. performed a retrospective study in 190 postmenopausal patients with symptomatic endometrial cancer and 16 asymptomatic patients showing no difference in survival in this relatively small series [7]. Osmer et al. compared 61 and 22 postmenopausal women with symptomatic and asymptomatic endometrial cancer, respectively. Patients with asymptomatic endometrial cancers showed a less myometrial tumor infiltration and more well differentiated tumors than symptomatic patients. A better prognosis for asymptomatic patients has been suggested [8]. Kimura et al. reviewed a total of 304 endometrial cancer patients [9]. Patients diagnosed with asymptomatic endometrial cancer showed a significantly better 5-year overall survival rate than the patients diagnosed after the onset of postmenopausal bleeding. The distribution of clinical stages and histological grades did not differ between both groups.

The aim of the present study was to estimate whether asymptomatic patients with endometrial cancer have a better prognosis than symptomatic patients with endometrial cancer diagnosed after postmenopausal bleeding in a large series of postmenopausal patients with endometrial cancer in a multi-center study.

\section{Methods \\ Patients}

Clinical data were obtained retrospectively from files at the Medical University of Vienna, Department of Obstetrics and Gynecology, the Landeskrankenhaus Klagenfurt,
Department of Obstetrics and Gynecology, and the Innsbruck Medical University, Department of Obstetrics and Gynecology. Six hundred five consecutive patients (Medical University of Vienna: $\mathrm{n}=388$, Landeskrankenhaus Klagenfurt: $\mathrm{n}=79$, Innsbruck Medical University: $\mathrm{n}=138$ ) with histological confirmed endometrial cancer undergoing surgery between December 1995 and January 2005 were included in our study. Five hundred forty three patients with endometrioid endometrial cancer were enrolled in all further analysis. Other histological subtypes were excluded from further analysis.

Based on patients' history and on the clinical examination performed by the referring physician and at time of referral to hospital, patients were classified into two groups according to presence (symptomatic patients) or absence (asymptomatic patients) of postmenopausal bleeding. Asymptomatic patients were defined as neglecting a type of bleeding in the previous 12 months, as having no signs of bleeding seen during the gynecological examination performed by the referring physician and at time of referral to hospital, and as not having any other symptoms indicative for endometrial cancer. Patients' personal history has been performed in the participating study centers in a standardized way. One of the most important questions was presence/absence of postmenopausal bleeding. This has been clearly documented in all patients' files.

\section{Clinical management}

A guideline by the Austrian Society of Gynecology and Obstetrics (OEGGG) and the Austrian Society of Gynecologic Oncology (AGO) recommends endometrial sampling in cases of a postmenopausal bleeding or an endometrial thickness $>11 \mathrm{~mm}$ in asymptomatic women [10]. Asymptomatic women included in our study are real asymptomatic cases detected by "quasi-screening" and did not seek care for other reasons. Diagnosis of endometrial cancer was established by hysteroscopy, dilatation and curettage. Subsequently, patients were treated by hysterectomy, bilateral salping-oophorectomy, pelvic and in selected cases paraaortic lymphadenectomy. In cases of lymph node metastases, postoperative radiotherapy was applied according to standardized treatment protocols. Histological staging and grading was performed according to the current International Federation of Gynecology and Obstetrics (FIGO) classification on basis of the final pathologic evaluation of hysterectomy specimen.

Patients were followed up in regular intervals after surgery, including inspection, vagino-rectal and screening for serum tumor marker evaluation. In cases of clinically suspicious findings and/or tumor marker elevation computed tomography was performed. The same protocols for treatment and follow-up were used in all participating centers. 
Table I: Patients' characteristics.

\begin{tabular}{|c|c|c|c|}
\hline Parameter & Asymptomatic patients (No.[\%]) & Symptomatic patients (No. [\%]) & $\mathbf{P}$ \\
\hline Number of patients & 149 & 456 & \\
\hline Patients with endometrioid endometrial cancer & 133 & 410 & \\
\hline Age at first diagnosis (years SD ) & $66.3(10.3)$ & $67.1(11.2)$ & $0.9 *$ \\
\hline Tumor stage & & & $0.07 \dagger$ \\
\hline 1 & $100(75.2)$ & $286(69.8)$ & \\
\hline II & $9(6.8)$ & $40(9.8)$ & \\
\hline III & $15(11.2)$ & $71(17.2)$ & \\
\hline IV & $9(6.8)$ & $13(3.2)$ & \\
\hline Tumor grade & & & $0.006+$ \\
\hline GI & 74 (55.7) & $170(41.5)$ & \\
\hline G2 & $45(33.8)$ & $153(37.3)$ & \\
\hline G3 & $14(10.5)$ & $87(21.2)$ & \\
\hline Patients with follow-up information available & 133 & 409 & \\
\hline Time of follow-up (months [SD]) & $23.7(24.03)$ & $28.5(25.76)$ & $0.06 *$ \\
\hline \multicolumn{4}{|l|}{ Recurrence status } \\
\hline Patients with recurrent disease & $14(10.5)$ & $55(13.4)$ & $0.2+$ \\
\hline Time to recurrent disease (months [SD]) & $18.5(16.9)$ & $20.4(17.6)$ & $0.7^{*}$ \\
\hline Status at last observation & & & $0.7 \dagger$ \\
\hline Alive with no evidence of disease & II $15(86.5)$ & $34 \mid(83.4)$ & \\
\hline Stable disease & 0 & $3(0.7)$ & \\
\hline Progressive disease & $8(6.0)$ & $34(8.3)$ & \\
\hline Dead as a result of disease & $9(6.8)$ & $25(6.1)$ & \\
\hline Dead as a result of other causes & $\mathrm{I}(0.7)$ & $6(1.5)$ & \\
\hline
\end{tabular}

$\mathrm{SD}=$ standard deviation, $*$ two sided student's t-test, $\dagger$ Chi squared test

\section{Statistical analysis}

Values are given as means (standard deviation [SD]) for evenly distributed values. Metric parameters were compared using Student's t-test. All data a Student's t-test was used for, were normally distributed. Nominal parameters were compared using Chi square test. Survival probabilities were calculated by a univariable Cox regression analysis (metric variables) or a product limit method of Kaplan and Meier using the log-rank test (categorical variables). A multivariable Cox regression model was performed comprising tumor stage (FIGO I vs. FIGO II vs. FIGO III vs. FIGO IV), tumor grade (G1 vs. G2 vs. G3), age at diagnosis, and presence/absence of irregular bleeding. The results were analyzed for the endpoint of disease-free and overall survival. Survival times of patients disease-free or still alive were censored with the last follow-up date. The proportional hazards assumption has been checked and found not to be violated. P-values of $<0.05$ were considered statistically significant. We used the statistical soft- ware SPSS 11.0 for Windows (SPSS 11.0, SPSS Inc., Chicago, IL) for statistical analysis.

\section{Institutional review board}

The present study was approved by our institutional review board, the Ethics-Committee of the Medical University of Vienna and the Vienna General Hospital - AKH.

\section{Results}

Patients' characteristics of asymptomatic/symptomatic patients with endometrial cancer are shown in Table 1. Asymptomatic patients with endometrial cancer had a significantly higher rate of well differentiated tumors. FIGO tumor stages and patients' age were evenly distributed. In univariable and multivariable survival analysis, tumor stage, tumor grade, and patients' age at diagnosis were associated with disease free and overall survival (Table 2, Table 3). In a multivariable survival analysis presence/ absence of a postmenopausal bleeding prior to diagnosis

Table 2: Univariable survival analysis in patients with endometrial cancer.

\begin{tabular}{|c|c|c|c|c|}
\hline & \multicolumn{2}{|c|}{ Disease free survival } & \multicolumn{2}{|c|}{ Overall survival } \\
\hline & HR (95\% CI) & $\mathbf{P}$ & HR (95\% CI) & $\mathbf{P}$ \\
\hline Tumor stage (FIGO I vs. II vs. III vs. IV) & - & $<0.001 *$ & - & $<0.001 *$ \\
\hline Tumor grade (GI vs. G2 vs. G3) & - & $<0.001 *$ & - & $<0.00 I^{*}$ \\
\hline Age at diagnosis & $1.03(1.01-1.05)$ & $0.003 t$ & $1.05(1.03-1.08)$ & $<0.001 \dagger$ \\
\hline
\end{tabular}

* Kaplan-Meier analysis, † univariable Cox-Regression model, $\mathrm{HR}(95 \% \mathrm{Cl})=$ Hazard Ratio $(95 \%$ Confidence Interval) 
Table 3: Multivariable survival analysis in patients with endometrial cancer.

\begin{tabular}{|c|c|c|c|c|}
\hline & \multicolumn{2}{|c|}{ Disease free survival* } & \multicolumn{2}{|c|}{ Overall survival* } \\
\hline & $\mathrm{HR}(95 \% \mathrm{Cl})$ & $\mathrm{P}$ & $\mathrm{HR}(95 \% \mathrm{Cl})$ & $P$ \\
\hline Tumor stage (FIGO I vs. II vs. III vs. IV) & $1.9(1.5-2.5)$ & $<0.001$ & $2.2(1.7-2.8)$ & $<0.001$ \\
\hline Tumor grade (G1 vs. G2 vs. G3) & $1.8(1.3-2.6)$ & 0.001 & $1.6(1.1-2.4)$ & 0.008 \\
\hline Age at diagnosis & $1.03(1.01-1.06)$ & 0.01 & $1.05(1.03-1.08)$ & $<0.001$ \\
\hline Bleeding vs. non bleeding & $0.9(0.5-1.6)$ & 0.7 & $0.8(0.5-1.4)$ & 0.4 \\
\hline
\end{tabular}

$*$ multivariable Cox-Regression model, HR $(95 \% \mathrm{Cl})=$ Hazard Ratio $(95 \%$ Confidence Interval)

was not associated with disease free or overall survival (Table 3). Kaplan-Meier curves for the influence of presence/absence of a postmenopausal bleeding on diseasefree (Figure 1) and overall (Figure 2) survival are shown.

\section{Discussion}

We present data on the prognostic value of presence/ absence of a postmenopausal bleeding prior to diagnosis in large series of patients with endometrial cancer in a multi-center study. In parallel to previous studies [9], we did not ascertain a significant difference in prognosis between asymptomatic and symptomatic patients.

Screening for gynecological malignancies has been advocated in order to diagnose diseases at earlier stage leading to earlier treatment with less side-effects and better prognosis [11]. To date, screening tools for endometrial cancer

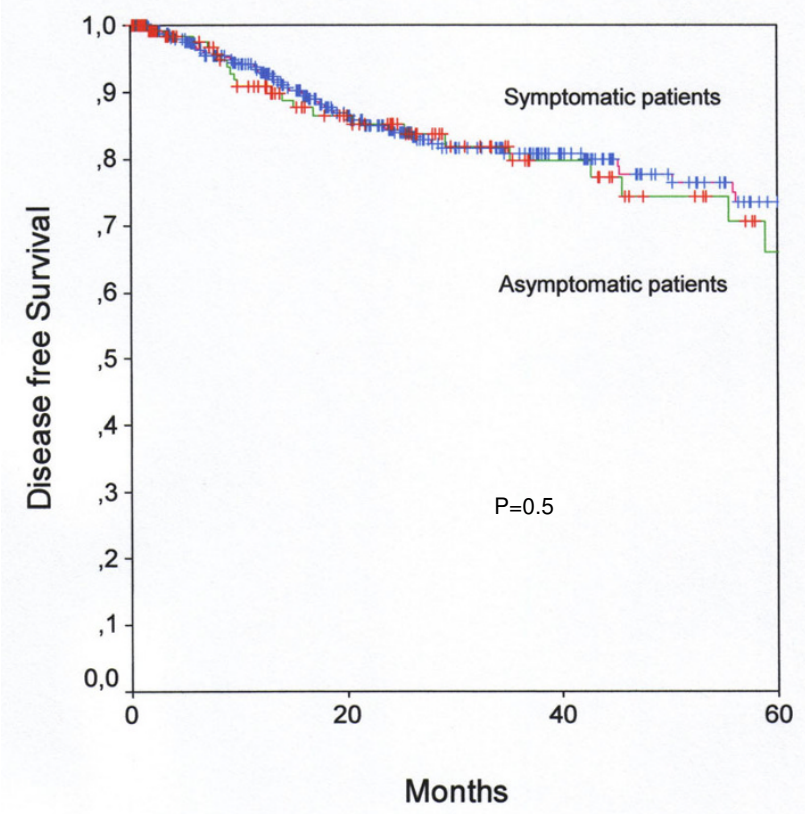

Figure I

Disease free survival. Kaplan-Meier curve for disease free survival of asymptomatic and symptomatic patients with endometrioid endometrial cancer. have not been identified. Transvaginal ultrasound has been suggested to detect endometrial cancer in an earlier stage before the onset of clinically recognizable symptoms [6]. Our study falls short of showing any statistically significant difference in disease-free and overall survival of affected patients.

Based on our study design we cannot extrapolate our results to a real screening situation. Although having no formally organized screening program in Austria, the majority of postmenopausal women routinely see their gynecologist once a year, i.e. opportunistic screening. Besides the PAP smear, a transvaginal sonography is routinely performed during this examination. Based on the currently available evidence [5], a guideline by the Austrian Society of Gynecology and Obstetrics (OEGGG) and the Austrian Society of Gynecologic Oncology (AGO) rec-

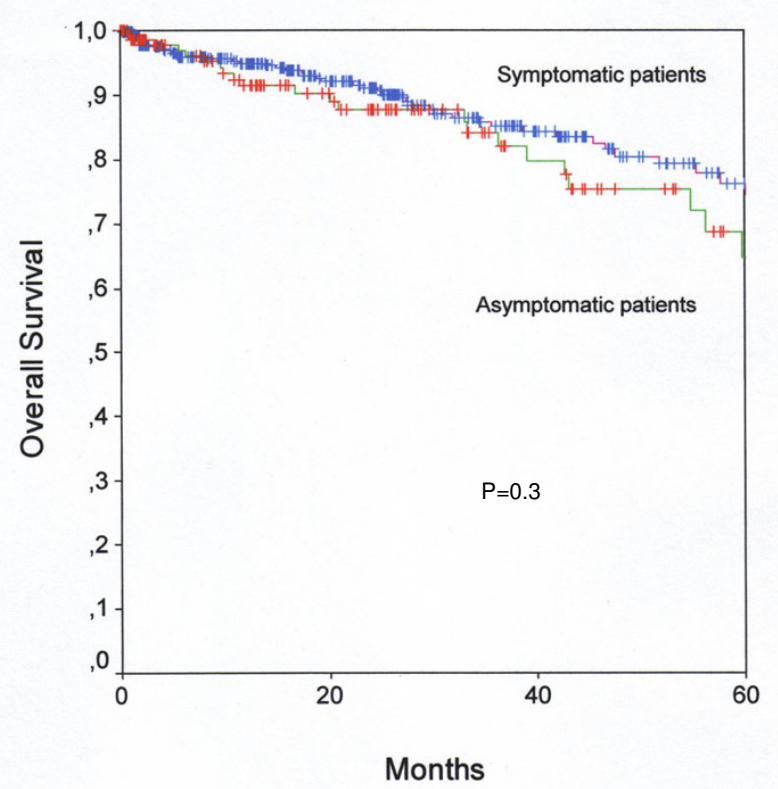

Figure 2

Overall Survival. Kaplan-Meier curve for overall survival of asymptomatic and symptomatic patients with endometrioid endometrial cancer. 
ommends endometrial sampling in cases of asymptomatic women and an endometrial thickness > $11 \mathrm{~mm}$ [10]. This might be the most likely reason for having only $3 / 4$ of women with endometrial cancer presenting with postmenopausal bleeding.

Of note, our study was not set out to evaluate the advantages or disadvantages of a transvaginal ultrasound in the screening of endometrial cancer. Studies demonstrating a positive effect of performing routine endometrial cancer screening on overall mortality would possibly have to include hundreds of thousands of women based on the relatively low incidence of the disease, the high rate of symptomatic women in early stage disease, and the good prognosis of affected women. Due to high costs and missing data on the reliability of TVS as screening tool for endometrial cancer the realization of a study like this would be hard to justify $[12,13]$. We speculate that such large-scale studies will not be performed and completed in the near future.

Our study supports previously published studies indicating that tumor biology in asymptomatic patients was different than in symptomatic patients [8]. This possibly reflects a higher propensity of low-differentiated tumors to cause postmenopausal bleeding than well/moderate differentiated endometrial cancers.

Our study has strengths and weaknesses, namely the retrospective study design, the extraction of information from patients' files, the relatively low number of events due to a generally good prognosis of affected patients, and the relatively short time of follow up. Another interesting point to mention is the relatively high percentage of women having advanced FIGO stages, i.e., FIGO II-IV, and still not having any symptoms. This can be easily explained in patients with FIGO IIIA, but not for other stages. Of note, we double-checked all FIGO stages and found our numbers to be correct. This has to be considered when interpreting the results of our study.

\section{Conclusion}

To conclude, asymptomatic patients with endometrial cancer undergoing surgery have a higher rate of well and moderate differentiated tumors compared to patients with a postmenopausal bleeding prior to diagnosis. The prognosis of both groups of patients was similar.

\section{Competing interests}

The authors declare that they have no competing interests.

\section{Authors' contributions}

V.S. initiated the design of the study with L.H. and A.R. and was involved in developing and conducting the study, wrote drafts of the paper and reviewed the manuscript.
M.S., K.H-F., H.L. and N.C. obtained the data from medical records, prepared the data for analysis and participated in reviewing of the manuscript. S.P. carried out statistical analysis and participated in reviewing the manuscript. A.R. and L.H. initiated the study design with V.S., coordinated analysis, interpreted results and participated in the writing and reviewing of the paper. All authors have read and approved the final manuscript.

\section{References}

I. Jemal A, Siegel R, Ward E, Murray T, Xu J, Thun MJ: Cancer statistics, 2007. CA Cancer J Clin 2007, 57:43-66.

2. Bokhman JV: Two pathogenetic types of endometrial carcinoma. Gynecol Oncol 1983, 15:10-7.

3. American College of Obstetricians and Gynecologists: ACOG practice bulletin, clinical management guidelines for obstetrician-gynecologists, number 65, August 2005: management of endometrial cancer. Obstet Gynecol 2005, 106:413.

4. Gu M, Shi W, Barakat RR, Thaler HT, Saigo PE: Pap smears in women with endometrial carcinoma. Acta Cytol 200I, 45:555-60.

5. Smith-Bindman R, Weiss E, Feldstein V: How thick is too thick? When endometrial thickness should prompt biopsy in postmenopausal women without vaginal bleeding. Ultrasound Obstet Gynecol 2004, 24:558-65.

6. Smith RA, von Eschenbach AC, Wender R, Levin B, Byers T, Rothenberger D, et al: American Cancer Society guidelines for the early detection of cancer: update of early detection guidelines for prostate, colorectal, and endometrial cancers. Also: update $200 \mathrm{I}$--testing for early lung cancer detection. CA Cancer J Clin 200I, 5 I:38-75.

7. Gerber B, Krause A, Muller H, Reimer T, Kulz T, Kundt G, et al.: Ultrasonographic detection of asymptomatic endometrial cancer in postmenopausal patients offers no prognostic advantage over symptomatic disease discovered by uterine bleeding. Eur J Cancer 200 I, 37:64-7I.

8. Osmers RG, Osmers M, Kuhn W: Prognostic value of transvaginal sonography in asymptomatic endometrial cancers. Ultrasound Obstet Gynecol 1995, 6:103-7.

9. Kimura T, Kamiura S, Yamamoto T, Seino-Noda H, Ohira H, Saji F: Abnormal uterine bleeding and prognosis of endometrial cancer. Int J Gynaecol Obstet 2004, 85: I 45-50.

10. Petru E, Sevelda P, Reinthaller A, Denison U, Wildt L, Lahousen M, Staudach A: Evaluation of the endometrium in the asymptomatic patient. Guideline of the Austrian Society of Gynecology and Obstetrics (OEGGG) and the Austrian Society of Gynecologic Oncology (AGO). Version October 2006. [http://www.oeggg.at].

II. Avarette $\mathrm{H}$, Steren $A$, Nguyen $\mathrm{H}$ : Screening in gynecologic cancers. Cancer 1993, 72(3 Suppl): 1043-9.

12. Havrilesky L, Maxwell G, Myers E: Cost-effectiveness analysis of annual screening strategies for endometrial cancer. $\mathrm{Am} J$ Obstet Gynecol 2009, 200(6):. 640.e I-8. Epub 2009 Apr 19

13. Fleischer A, Wheeler J, Lindsay I, Hendrix S, Grabill S, Kravitz B, MacDonald $B$ : An assessment of the value of ultrasonographic screening for endometrial disease in postmenopausal women without symptoms. Am J Obstet Gynecol 200I, 184(2):70-5.

\section{Pre-publication history}

The pre-publication history for this paper can be accessed here:

http://www.biomedcentral.com/1471-2407/9/460/pre pub 